\title{
Review Article \\ Short-Term Effects of Verapamil and Diltiazem in the Treatment of No Reflow Phenomenon: A Meta-Analysis of Randomized Controlled Trials
}

\author{
Lan Wang, Zhong Cheng, Ye Gu, and Dingfeng Peng \\ Heart Center at Puai Hospital, Wuhan Puai Hospital, Huazhong University of Science and Technology, Wuhan 430030, China
}

Correspondence should be addressed to Dingfeng Peng; dfpeng2003cn_puai@163.com

Received 28 July 2015; Revised 28 August 2015; Accepted 31 August 2015

Academic Editor: Kai Hu

Copyright (C) 2015 Lan Wang et al. This is an open access article distributed under the Creative Commons Attribution License, which permits unrestricted use, distribution, and reproduction in any medium, provided the original work is properly cited.

\begin{abstract}
Currently, there is still a lack of an optimal treatment for no reflow phenomenon (NRP). We analyzed the efficacy and safety of using nondihydropyridine calcium channel antagonists (NDHP, verapamil/diltiazem) in patients suffering from NRP. Eight RCTs with 494 participants were eligible for analysis. The pooling analysis showed that intracoronary verapamil/diltiazem injection significantly decreased the occurrence of the coronary NRP (RR: $0.3,95 \%$ CI: $0.16-0.57 ; P=0.0002$ ) and reduced corrected thrombolysis in myocardial infarction (TIMI) frame Count (WMD $=-9.24,95 \%$ CI $-13.91-4.57 ; P=0.0001)$ in patients with NRP. Moreover, verapamil/diltiazem treatment showed superiority in reducing wall motion index (WMI) compared to the control at day $1(\mathrm{WMD}=0.11,95 \% \mathrm{CI}: 0.02-0.20 ; P=0.02)(P<0.05)$. There was also a significantly greater decline at occurrence of the major adverse cardiac events between verapamil/diltiazem and control groups (WMD: 0.4, 95\% CI: 0.19-0.84; $P=0.02$ ). However, using verapamil/diltiazem did not provide additional improvement of left ventricular ejection fraction post procedure (at 7 days, WMD, 0.1; 95\% CI, $-2.43-2.63 ; P=0.94$; at 30 days, WMD, 0.42; 95\% CI, $-2.09-2.92 ; P=0.75)$. NDHP use is beneficial in attenuating NRP and reducing 6-month MACEs in patients with NRP.
\end{abstract}

\section{Introduction}

No-reflow phenomenon is defined as inadequate myocardial perfusion of the adequately dilated target vessel without evidence of angiographic mechanical obstruction [1], angiographic no flow refers to TIMI $0-1$ and slow flow refers to TIMI 2 [2], and no reflow and slow flow phenomenon have been observed in $0.6 \%$ to $2 \%$ of patients receiving percutaneous coronary interventions (PCIs) [3-6]. Incidence of angiographic no reflow phenomenon has been reported as high as $10 \%$ to $44 \%$ in acute myocardial infarction (AMI) patients undergoing PCI $[7,8]$. Clinical studies revealed that patients exhibiting no/slow reflow following reperfusion therapy ischemic heart disease were associated with worse prognosis compared to patients without no-flow $[4,6]$.

Key pathogenic components of no/slow flow phenomenon include distal atherothrombotic embolization, ischemic injury, reperfusion injury, and susceptibility of coronary microcirculation to injury [9]. Thus, pharmacologic and mechanical strategies to prevent and treat no reflow also target these mechanisms; pharmacologic therapy with vasodilators and antiplatelet agents as well as mechanical therapies with distal protection and aspiration thrombectomy have shown benefit in the treatment of no/slow reflow phenomenon [10]. Although local vasodilator therapy and local antiplatelet therapy are widely used to manage the clinical no/slow phenomenon, only local vasodilator therapy has a specific guideline indication for treatment of no reflow and The 2011 ACC PCI guidelines [11] give a class IIa recommendation for administration of an intracoronary vasodilator (specifically, adenosine, calcium channel blocker, or nitroprusside) to treat PCI-related no reflow that occurs during primary or elective PCI.

Efficacy of intracoronary nondihydropyridine calcium channel antagonists (NDHP), verapamil/diltiazem, administration during PCI for the management of reduced coronary flow due to microvascular dysfunction was already evidenced in the 1990s in selected patients $[12,13]$. Previous studies 


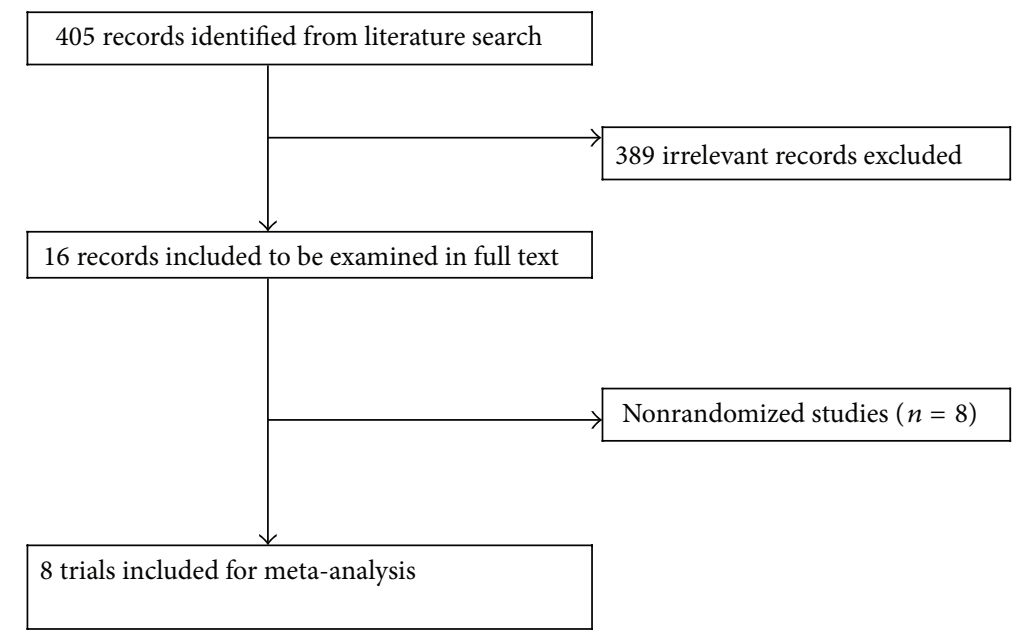

FIGURE 1: Flow chart of trials selection. Flow chart shows the literature search for RCTs of NDHP versus control group for patients with slow flow or no reflow. RCTs indicate randomized controlled trial.

also showed that NDHP significantly improved coronary and myocardial perfusion when given prophylactically prior to elective PCI $[14,15]$ and in patients undergoing primary PCI for AMI [16]. Recently, Su et al. analyzed the short-term effect of verapamil [17] as well as adenosine and verapamil [18] on coronary no reflow associated with percutaneous coronary intervention in patients with acute coronary syndrome. Present meta-analysis focused on the efficacy and safety of verapamil/diltiazem use for the management of no/slow phenomenon.

\section{Methods}

2.1. Search Strategy. We search the PubMed, OVID, EBSCO, and Cochrane Library (1980-2015) to identify randomized controlled trials (RCTs) and observational studies. Searches were conducted without any language restrictions using the key words "slow flow," "no reflow," "verapamil," and "diltiazem".

2.2. Study Selection. The selection criteria for inclusion in this meta-analysis were as follows: RCTs studies that randomly allocated patients with coronary no reflow or slow flow to undergo intracoronary/oral infusion of verapamil/diltiazem or placebo/blank control; studies reported at least on one of following outcomes: thrombolysis in myocardial infarction (TIMI) flow as well as TIMI frame count (CTFC), major adverse cardiac events (MACEs, such as all-cause death, target vessel revascularization, recurrent angina or myocardial infarction, and severe heart failure), left ventricular ejection fraction (LVEF), or wall motion index (WMI).

2.3. Data Extraction. Data extraction was performed by two independent reviewers using a standardized data-collection form, and disagreements were resolved by discussion. Patient characteristics, study quality, and clinical outcomes including CTFC, TIMI flow, MACEs, LEVF, and WMI were analyzed in both NDHP and control groups.
2.4. Methodological Quality Assessment. We evaluated trials for concealment of treatment allocation, clear description of the design, and completeness of follow-up. The JADAD scale [26] was used to score study quality (range of 0-5, higher scores indicating higher quality). Quality assessment was undertaken independently by two reviewers.

2.5. Statistical Analysis. Results were analyzed with Review Manager 5.2 software (Cochrane Collaboration, Oxford, England). Egger's asymmetry test was performed using Stata13.0 software (available from http://stata.com/). Heterogeneity was tested using the $\chi^{2}$ test (with $P<0.05$ indicating significant heterogeneity). The meta-analysis was performed using the fixed effects model if $P>0.05$; otherwise, the random effect model was used. Odds ratios (ORs) were calculated for dichotomous variables and the weighted mean difference (WMD) was calculated for continuous variables. Summary statistics were reported with 95\% confidence intervals (CIs). Publication bias was assessed by visual examination of the funnel plots and by using Egger's asymmetry test.

\section{Results}

3.1. Included Studies and Patient Characteristics. We initially identified 405 potentially relevant articles and 16 articles were considered to be of interest and were retrieved for full-text review. Eight non-RCTs studies were excluded, and the remaining 8 RCTs studies [16, 19-25] were analyzed. Figure 1 shows the flow chart of the study selection process. Of the 8 articles, one article [20] reported the results of the RECOVER trial by comparison of three groups (verapamil group, diltiazem group, and control group); we analyzed these data by dividing the data sets into verapamil versus control and diltiazem versus control. The 8 published RCTs included a total of 494 patients (261 allocated to NDHP and 233 to the control group). Study quality was generally lowto-intermediate (three studies with JADAD scale 2, three studies with JADAD scale 3 , and only one study with JADAD 
TABLE 1: Baseline characteristics of included studies.

\begin{tabular}{|c|c|c|c|c|c|c|c|c|}
\hline \multirow{2}{*}{ Study } & \multirow{2}{*}{ Year } & \multirow{2}{*}{$\begin{array}{c}N \\
\text { (Ver.) }\end{array}$} & \multirow{2}{*}{$\begin{array}{c}N \\
\text { (Dil.) }\end{array}$} & \multirow{2}{*}{$\begin{array}{c}N \\
\text { (Con.) }\end{array}$} & \multicolumn{2}{|c|}{ Interventions } & \multirow{2}{*}{ Outcomes } & \multirow{2}{*}{ Jadad score } \\
\hline & & & & & Expt. & Ctrl. & & \\
\hline $\begin{array}{l}\text { Taniyama et al. } \\
{[16]}\end{array}$ & 1997 & 20 & 0 & 20 & $\begin{array}{l}0.5 \mathrm{mg} \text { verapamil } \\
\text { (diluted in } 10 \mathrm{~mL} \\
\text { saline) } \\
120 \text { mg po follow-up } \\
\text { study }\end{array}$ & Blank & TIMI/LVEF/WMI & 3 \\
\hline $\begin{array}{l}\text { Vijayalakshmi } \\
\text { et al. [19] }\end{array}$ & 2006 & 49 & 0 & 50 & $0.5 \mathrm{mg}$ verapamil & Heparinized & TIMI/MACE/TFC/WMI & 3 \\
\hline $\begin{array}{l}\text { Hendler et al. } \\
\text { [20] }\end{array}$ & 2006 & 10 & 0 & 10 & $0.5 \mathrm{mg}$ verapamil & Heparinized & TIMI/LVEF & 2 \\
\hline $\begin{array}{l}\text { Chang et al. } \\
\text { [21] }\end{array}$ & 2010 & 29 & 0 & 35 & $100-400 \mu \mathrm{g}$ verapamil & Nitroglycerin & CTFC & 2 \\
\hline $\begin{array}{l}\text { Huang et al. } \\
\text { [22] }\end{array}$ & 2012 & 34 & 34 & 34 & $\begin{array}{c}200 \mu \mathrm{g} \text { verapamil (max } \\
1 \mathrm{mg}) \\
400 \mu \mathrm{g} \text { diltiazem (max } \\
1 \mathrm{mg})\end{array}$ & $\begin{array}{l}\text { Nitroglycerin } \\
\text { (max } 1 \mathrm{mg})\end{array}$ & TIMI/CTFC/LVEF/MACE & 3 \\
\hline Li et al. [23] & 2012 & 0 & 40 & 39 & $\begin{array}{c}\text { Diltiazem } 90 \mathrm{mg} \text { bid/6 } \\
\text { months }\end{array}$ & Placebo & TIMI/CTFC/LVEF/MACE & 6 \\
\hline $\begin{array}{l}\text { Ozdogru et al. } \\
{[24]}\end{array}$ & 2013 & 0 & 30 & 30 & $5 \mathrm{mg}$ diltiazem & Nitroglycerin & CTFC/HR/BP & 2 \\
\hline $\begin{array}{l}\text { Akturk et al. } \\
\text { [25] }\end{array}$ & 2014 & 15 & 0 & 15 & Verapamil & Placebo & CTFC/LEVF & 2 \\
\hline Overall & & 157 & 104 & 233 & & & & \\
\hline
\end{tabular}

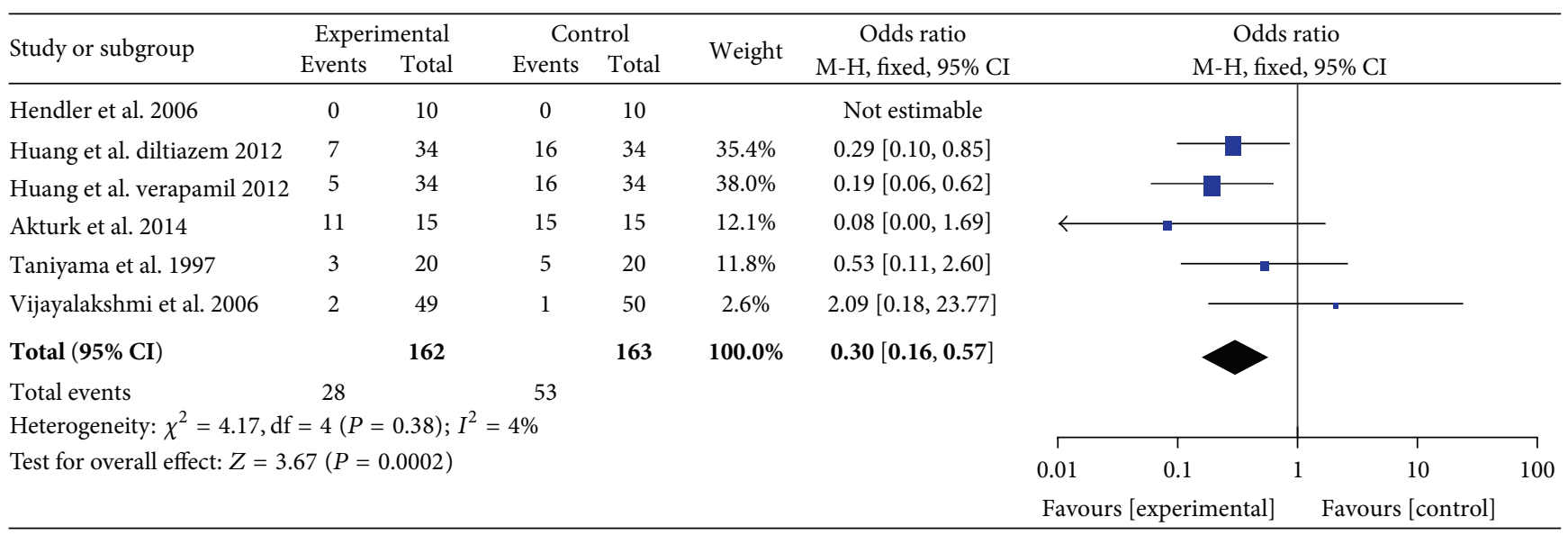

FIGURE 2: Pooled risk ratio of verapamil/diltiazem versus control for TIMI flow after percutaneous coronary intervention. CI, confidence interval; M-H, Mantel-Haenszel; TIMI, thrombolysis in myocardial infarction.

scale 6). Table 1 shows the study design and baseline patient characteristics for each of the eligible trials. No significant differences were found in baseline characteristics between the 2 groups. The mean follow-up period ranged from 1 to 6 months.

\subsection{Outcomes}

3.2.1. TIMI Flow. Data on TIMI flow were available from five studies $[16,19,20,22,25]$ randomizing 325 participants, of whom 162 received verapamil/diltiazem therapy and 163 received control therapy. The pooling analysis showed that intracoronary verapamil/diltiazem injection significantly decreased the occurrence of the coronary no reflow phenomenon (RR: $0.3,95 \% \mathrm{CI}$ : 0.16 to $0.57 ; P=0.0002, I^{2}=4 \%$; Figure 2).

3.2.2. CTFC. Data on CTFC were available from six data sets derived from five studies [21-25] randomizing 360 participants. Intracoronary verapamil/diltiazem injection or oral diltiazem significantly decreased the CTFC as compared to control $(\mathrm{WMD}=-9.24,95 \% \mathrm{CI}-13.91$ to $4.57 ; P=0.0001$; $I^{2}=73 \%$, Figure 3 ). 


\begin{tabular}{lccccccccc|c}
\hline \multirow{2}{*}{ Study or subgroup } & \multicolumn{9}{c}{ Experimental } & \multicolumn{3}{c}{ Control } & \multicolumn{4}{c}{ Mean difference } & \multicolumn{2}{c}{ Mean difference } \\
IV, random, 95\% CI
\end{tabular}

Figure 3: Pooled mean difference of verapamil/diltiazem therapy versus control for correct thrombolysis in myocardial infarction frame count after percutaneous coronary intervention. CI, confidence intervals; IV, inverse variance; SD, standard deviation.

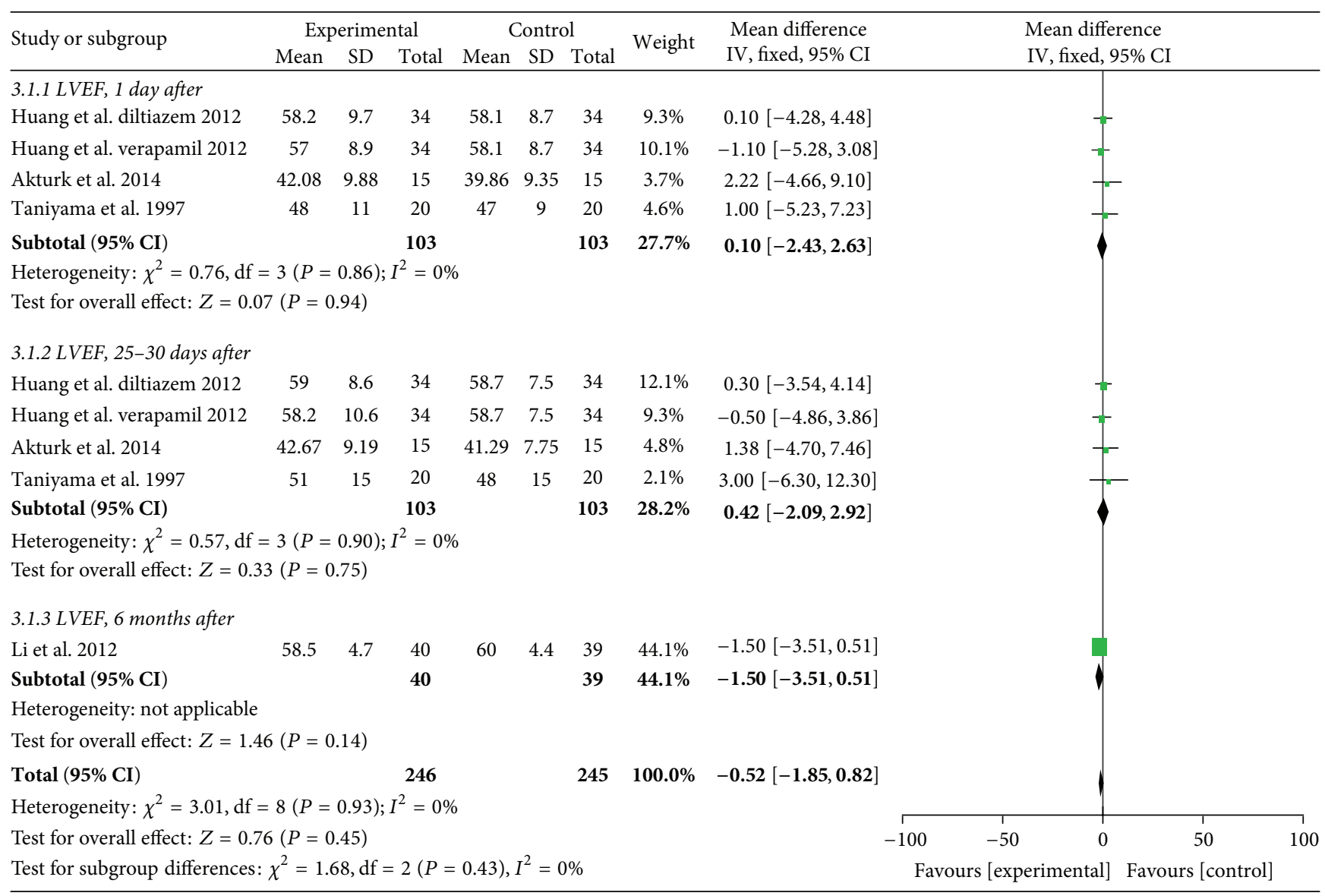

FIGURE 4: Pooled mean difference of experimental versus control group for left ventricular ejection fraction (LVEF) after percutaneous coronary intervention (PCI). CI, confidence intervals; IV, inverse variance; SD, standard deviation.

3.2.3. $L V E F$. Three RCTs $[16,22,23]$ reported $L V E F$ values and there was no significant difference on LVEF between NDHP and control groups within one week (WMD, 0.1; 95\% CI, -2.43 to $2.63 ; P=0.94 ; I^{2}=0 \%$, Figure 4$)$, and after 30 days (WMD, $0.42 ; 95 \% \mathrm{CI},-2.09$ to $2.92 ; P=0.75 ; I^{2}=0 \%$, Figure 4). One study [25] observed no difference on LVEF between baseline and 6 months after therapy between oral diltiazem group and control group $(P>0.05$, Figure 4$)$.

3.2.4. WMI. WMI data were available from two studies $[16,19]$; verapamil/diltiazem treatment showed superiority in reducing WMI compared to the control at day $1(P<0.05$, 


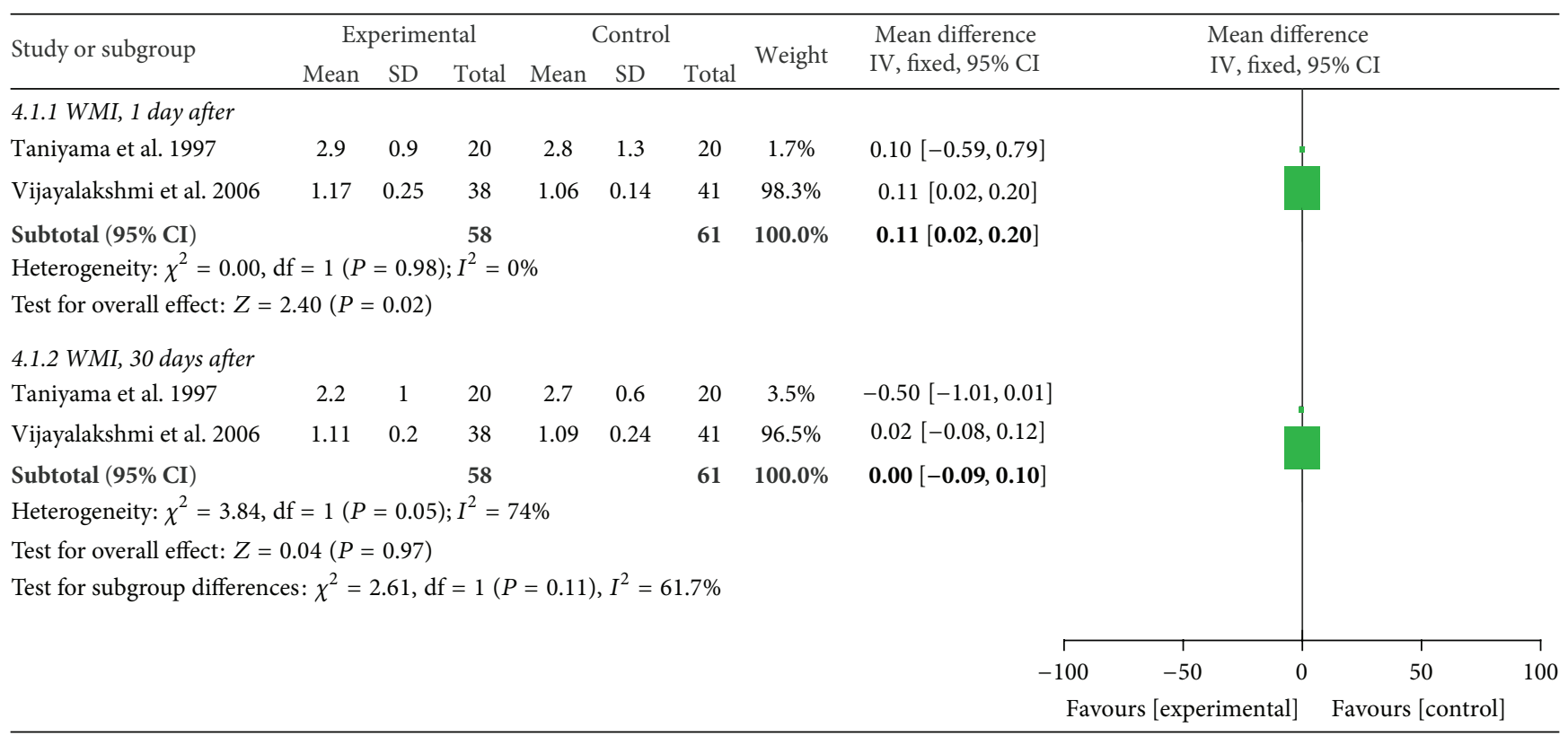

FIGURE 5: Pooled risk ratio of experimental therapy versus control for WMI after percutaneous coronary intervention. CI, confidence interval; M-H, Mantel-Haenszel.

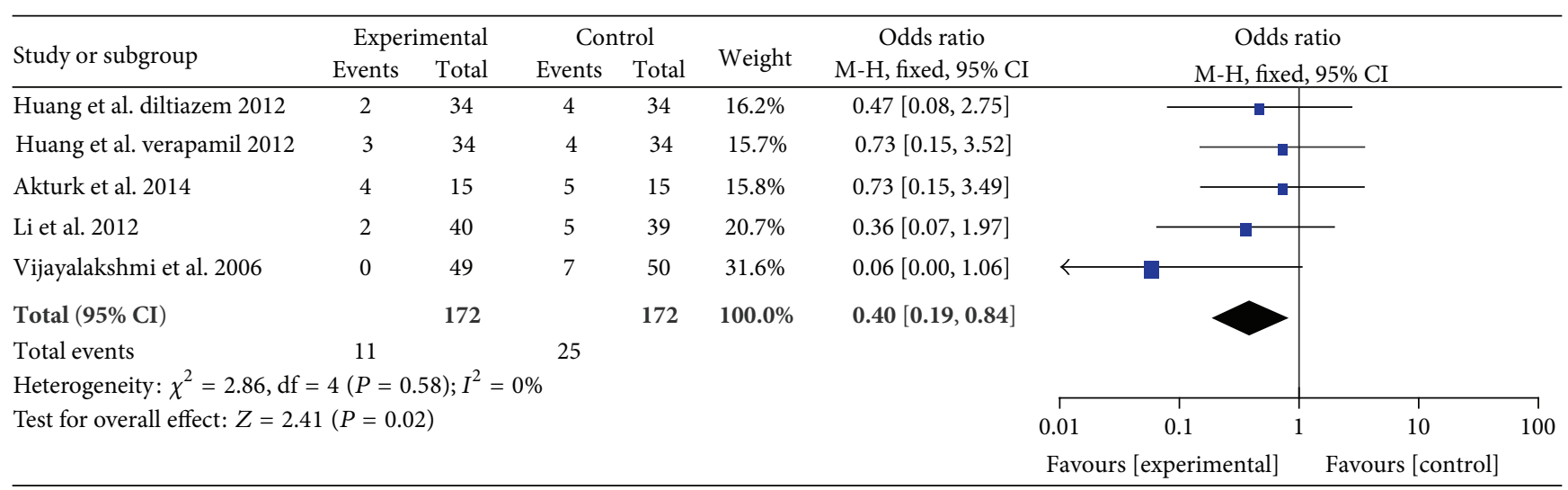

FIGURE 6: Pooled risk ratio of experimental therapy versus control for major adverse cardiac events after percutaneous coronary intervention. $\mathrm{CI}$, confidence interval; M-H, Mantel-Haenszel.

Figure 5). However, there was no significant difference at day 30 after therapy $(P>0.05$, Figure 5$)$.

3.2.5. MACEs. Five experimental data from four RCTs [19, 22, 23, 25] reported the MACEs. Incidence of MACEs was significantly lower in intracoronary verapamil/diltiazem injection or oral diltiazem groups compared to control group at 6 months after PCI (WMD: $0.4,95 \%$ CI: 0.19 to $0.84 ; P=$ $0.02 ; I^{2}=0 \%$, Figure 6$)$.

3.3. Publication Bias. There is no evidence of publication bias for studies included in this meta-analysis based on the Egger's test $(P=0.308)$.
3.4. Side Effects. Side effects were not reported in 3 RCTs $[20,21,25]$. No side effects were observed in one study [16]. Vijayalakshmi et al. reported transient blood pressure reduction and heart block up to $3 \mathrm{~h}$ after intracoronary verapamil injection in nine patients [19]. Huang and colleagues also observed transient blood pressure reduction $(9 \mathrm{mmHg})$ and bradycardia in patients after intracoronary verapamil injection ( 4 sinus bradycardia and 5 cases with $\mathrm{AVB} \geq 2,4$ out of 5 patients received pacemaker implantation [22]). Li et al. reported reduced heart rate after oral diltiazem treatment [23] and Ozdogru et al. reported heart rate and blood pressure reduction after intracoronary verapamil injection [24]. In general, NDHP use is safe, but heart rate and blood pressure monitoring are essential during NDHP therapy to observe 
and timely treat the bradycardia and blood pressure reduction when indicated.

\section{Discussion}

Despite intense research in the field of NRP, no single mechanical or pharmacological therapy has demonstrated a clear efficacy against NRP, due to its multifactorial nature [27]. Results from present meta-analysis indicate that intracoronary verapamil/diltiazem injection or oral diltiazem is associated with a significantly improved TIMI flow and CTFC and reduced incidence of short-term MACEs in patients with no/slow flow. In addition, the procedure significantly reduced WMI compared to the control group. However, verapamil/diltiazem treatment did not affect cardiac systolic function as shown by similar LVEF between NDHP and control group.

Verapamil/diltiazem, nondihydropyridine calcium channel blocker, is known to block the L-type channel in the cell membrane and decrease heart rate, the rate of atrioventricular conduction, blood pressure, and myocardial contractility [28, 29]. However, verapamil/diltiazem treatment did not affect cardiac systolic function as shown by similar LVEF between verapamil/diltiazem and control group based on data from this analysis. As a class, verapamil/diltiazem are beneficial in the prevention of the no reflow phenomenon that is thought to be related to their role in coronary vasodilation. Anatomic no reflow, as assessed by thioflavins [30], includes two different forms: structural and functional no reflow. In functional no reflow, patency of microvessels is compromised because of spasm and/or microembolisation instead of structural distintegrigy [31]. Alleviated coronary artery spasm, especially in the distal coronary artery, might be the major potential mechanism of intracoronary verapamil/diltiazem injection on attenuating the NRP [32].

\section{Clinical Implication}

Results from present analysis suggest that NDHP is uniquely effective in attenuating NRP and reducing short-term MACEs in NRP patients and these agents might be safely used in daily clinical work to combat the NRP. It is to note that NDHP use, especially the intracoronary verapamil injection, is sometimes related to blood pressure reduction and bradycardia; thus, blood pressure and heart rate monitoring are needed during the NDHP use. Future studies with large patient cohort are warranted to prove their efficacy and also in comparison with other potential effective medications currently used to manage NRP including antiplatelet therapy, adenosine, nicardipine, nicorandil, and sodium nitroprusside [27].

\section{Limitations}

This meta-analysis has several limitations. First, detailed description on the method of randomization and allocation concealment process of the 8 RCTs trials was not available; thus, we cannot exclude the existence of selection bias which might affect the clinical outcomes between the NDHP and control group. Second, examined parameters such as TIMI flow and CTFC are somehow objective indicators; the measurement bias might thus affect the reported results. Third, the Jadad score for the included 8 RCTs is not high because the treatment assignments are not double-blinded. Therefore, the results of the present analysis need to be interpreted with caution and may warrant further investigation with larger patient cohort and longer observation period.

\section{Conclusion}

NDHP (verapamil/diltiazem) use is safe and beneficial in attenuating NRP and reducing the 6-month MACEs in NRP patients.

\section{Conflict of Interests}

The authors declare that there is no conflict of interests regarding the publication of this paper.

\section{Authors' Contribution}

Lan Wang and Zhong Cheng contributed equally to this work.

\section{References}

[1] J. Schofer, R. Montz, and D. G. Mathey, "Scintigraphic evidence of the 'no reflow' phenomenon in human beings after coronary thrombolysis," Journal of the American College of Cardiology, vol. 5, no. 3, pp. 593-598, 1985.

[2] J. H. Chesebro, G. Knatterud, R. Roberts et al., "Thrombolysis in myocardial infarction (TIMI) trial, phase I: a comparison between intravenous tissue plasminogen activator and intravenous streptokinase. Clinical findings through hospital discharge," Circulation, vol. 76, no. 1, pp. 142-154, 1987.

[3] R. A. Kloner, C. E. Ganote, and R. B. Jennings, "The 'no reflow' phenomenon after temporary coronary occlusion in the dog," The Journal of Clinical Investigation, vol. 54, no. 6, pp. 1496-1508, 1974.

[4] R. N. Piana, G. Y. Paik, M. Moscucci et al., "Incidence and treatment of 'no-reflow' after percutaneous coronary intervention," Circulation, vol. 89, no. 6, pp. 2514-2518, 1994.

[5] K. M. Abbo, M. Dooris, S. Glazier et al., "Features and outcome of no-reflow after percutaneous coronary intervention," The American Journal of Cardiology, vol. 75, no. 12, pp. 778-782, 1995.

[6] R. A. Kloner, "No-reflow phenomenon: maintaining vascular integrity," Journal of Cardiovascular Pharmacology and Therapeutics, vol. 16, no. 3-4, pp. 244-250, 2011.

[7] H. Ito, A. Maruyama, K. Iwakura et al., "Clinical implications of the 'no reflow' phenomenon. A predictor of complications and left ventricular remodeling in reperfused anterior wall myocardial infarction," Circulation, vol. 93, no. 2, pp. 223-228, 1996.

[8] H. Ito and K. Iwakura, "Assessing the relation between coronary reflow and myocardial reflow," The American Journal of Cardiology, vol. 81, no. 12, supplement 1, pp. 8G-12G, 1998. 
[9] M. A. Chaudhry, M. Smith, E. B. Hanna, and R. Lazzara, "Diverse spectrum of presentation of coronary slow flow phenomenon: a concise review of the literature," Cardiology Research and Practice, vol. 2012, Article ID 383181, 6 pages, 2012.

[10] R. Berg and C. Buhari, "Treating and preventing no reflow in the cardiac catheterization laboratory," Current Cardiology Reviews, vol. 8, no. 3, pp. 209-214, 2012.

[11] G. N. Levine, E. R. Bates, J. C. Blankenship et al., “2011 ACCF/AHA/SCAI guideline for percutaneous coronary intervention. A report of the American College of Cardiology Foundation/American Heart Association Task Force on practice guidelines and the Society for Cardiovascular Angiography and Interventions," Journal of the American College of Cardiology, vol. 58, pp. e44-e122, 2011.

[12] R. M. Pomerantz, R. E. Kuntz, D. J. Diver, R. D. Safian, and D. S. Baim, "Intracoronary verapamil for the treatment of distal microvascular coronary artery spasm following PTCA," Catheterization and Cardiovascular Diagnosis, vol. 24, no. 4, pp. 283-285, 1991.

[13] F. J. Weyrens, J. Mooney, J. Lesser, and M. R. Mooney, "Intracoronary diltiazem for microvascular spasm after interventional therapy," The American Journal of Cardiology, vol. 75, no. 12, pp. 849-850, 1995.

[14] F. Jalinous, J. A. Mooney, and M. R. Mooney, "Pretreatment with intracoronary diltiazem reduces non-q-wave myocardial infarction following directional atherectomy," The Journal of Invasive Cardiology, vol. 9, no. 4, pp. 270-273, 1997.

[15] K. Saito, H. Nonogi, Y. Goto et al., "Antiischemic effect of intracoronary diltiazem on myocardial ischemia during PTCA," Heart and Vessels, vol. 11, no. 2, pp. 92-99, 1996.

[16] Y. Taniyama, H. Ito, K. Iwakura et al., "Beneficial effect of intracoronary verapamil on microvascular and myocardial salvage in patients with acute myocardial infarction," Journal of the American College of Cardiology, vol. 30, no. 5, pp. 1193-1199, 1997.

[17] Q. Su, L. Li, and Y. Liu, "Short-term effect of verapamil on coronary no-reflow associated with percutaneous coronary intervention in patients with acute coronary syndrome: a systematic review and meta-analysis of randomized controlled trials," Clinical Cardiology, vol. 36, no. 8, pp. E11-E16, 2013.

[18] Q. Su, T. S. Nyi, and L. Li, "Adenosine and verapamil for noreflow during primary percutaneous coronary intervention in people with acute myocardial infarction," Cochrane Database of Systematic Reviews, vol. 5, Article ID CD009503, 2015.

[19] K. Vijayalakshmi, V. J. Whittaker, B. Kunadian et al., "Prospective, randomised, controlled trial to study the effect of intracoronary injection of verapamil and adenosine on coronary blood flow during percutaneous coronary intervention in patients with acute coronary syndromes," Heart, vol. 92, no. 9, pp. 1278-1284, 2006.

[20] A. Hendler, A. Aronovich, E. Kaluski et al., "Optimization of myocardial perfusion after primary coronary angioplasty following an acute myocardial infarction. Beyond TIMI 3 flow," The Journal of Invasive Cardiology, vol. 18, no. 1, pp. 32-36, 2006.

[21] S.-F. Chang, J.-Y. Ma, J.-Y. Qian, X.-H. Shu, and J.-B. Ge, "Effects of intracoronary administration of nitroglycerin and verapamil for treatment of coronary slow flow phenomenon," Zhonghua Xin Xue Guan Bing Za Zhi, vol. 38, no. 1, pp. 27-30, 2010.

[22] D. Huang, J. Qian, L. Ge et al., "REstoration of COronary flow in patients with no-reflow after primary coronary interVEntion of acute myocaRdial infarction (RECOVER)," American Heart Journal, vol. 164, no. 3, pp. 394-401, 2012.
[23] L. Li, Y. Gu, T. Liu et al., "A randomized, single-center doubleblinded trial on the effects of diltiazem sustained-release capsules in patients with coronary slow flow phenomenon at 6month follow-up," PLoS ONE, vol. 7, no. 6, Article ID e38851, 2012.

[24] I. Ozdogru, C. Zencir, A. Dogan et al., "Acute effects of intracoronary nitroglycerin and diltiazem in coronary slow flow phenomenon," Journal of Investigative Medicine, vol. 61, no. 1, pp. 45-49, 2013.

[25] I. F. Akturk, A. A. Yalcin, I. Biyik et al., "Effects of verapamil and adenosine in an adjunct to tirofiban on resolution and prognosis of noreflow phenomenon in patients with acute myocardial infarction," Minerva Cardioangiologica, vol. 62, pp. 389-397, 2014.

[26] A. R. Jadad, R. A. Moore, D. Carroll et al., "Assessing the quality of reports of randomized clinical trials: is blinding necessary?" Controlled Clinical Trials, vol. 17, no. 1, pp. 1-12, 1996.

[27] P. Salinas, S. Jimenez-Valero, R. Moreno et al., "Update in pharmacological management of coronary no-reflow phenomenon," Cardiovascular and Hematological Agents in Medicinal Chemistry, vol. 10, no. 3, pp. 256-264, 2012.

[28] W. J. Elliott and C. V. S. Ram, "Calcium channel blockers," Journal of Clinical Hypertension, vol. 13, no. 9, pp. 687-689, 2011.

[29] V. Soukoulis, W. E. Boden, S. C. Smith, and P. T. O'Gara, "Nonantithrombotic medical options in acute coronary syndromes: old agents and new lines on the horizon," Circulation Research, vol. 114, no. 12, pp. 1944-1958, 2014.

[30] T. Reffelmann and R. A. Kloner, “The 'no-reflow' phenomenon: basic science and clinical correlates," Heart, vol. 87, no. 2, pp. 162-168, 2002.

[31] L. Galiuto, "Optimal therapeutic strategies in the setting of postinfarct no reflow: the need for a pathogenetic classification," Heart, vol. 90, no. 2, pp. 123-125, 2004.

[32] J.-L. Zhao, Y.-J. Yang, C.-J. Cui, S.-J. You, Y.-J. Wu, and R.-L. Gao, "Different effects of adenosine and calcium channel blockade on myocardial no-reflow after acute myocardial infarction and reperfusion," Cardiovascular Drugs and Therapy, vol. 20, no. 3, pp. $167-175,2006$. 


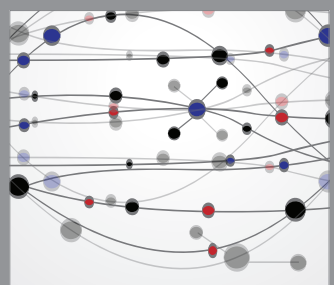

The Scientific World Journal
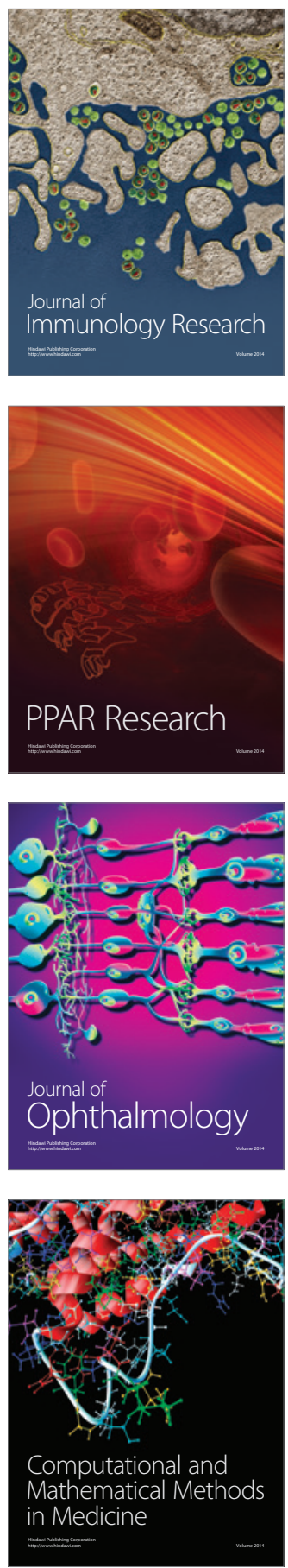

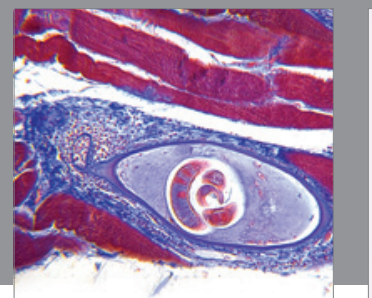

Gastroenterology

Research and Practice
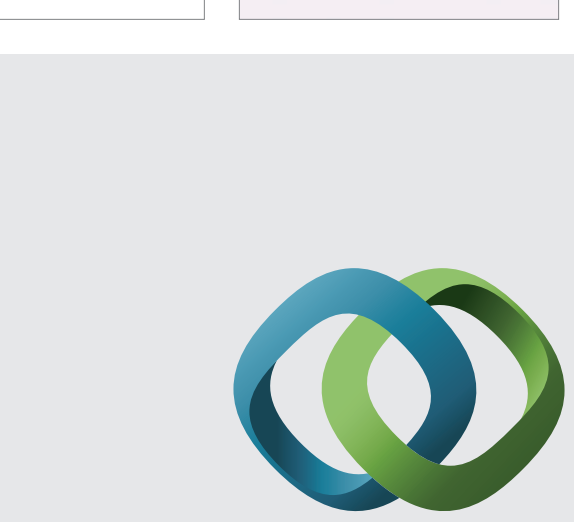

\section{Hindawi}

Submit your manuscripts at

http://www.hindawi.com
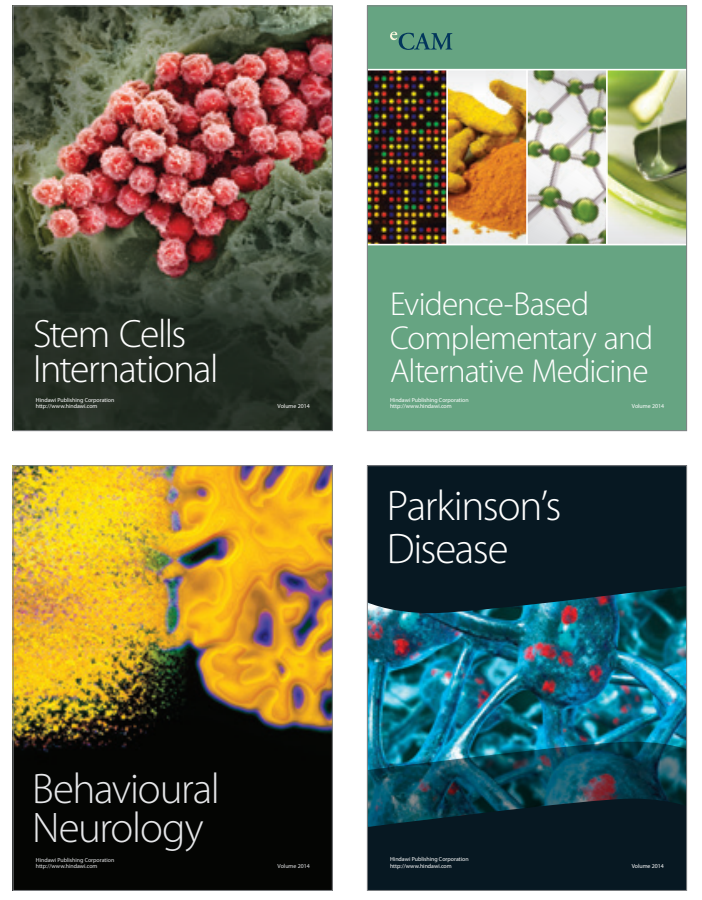
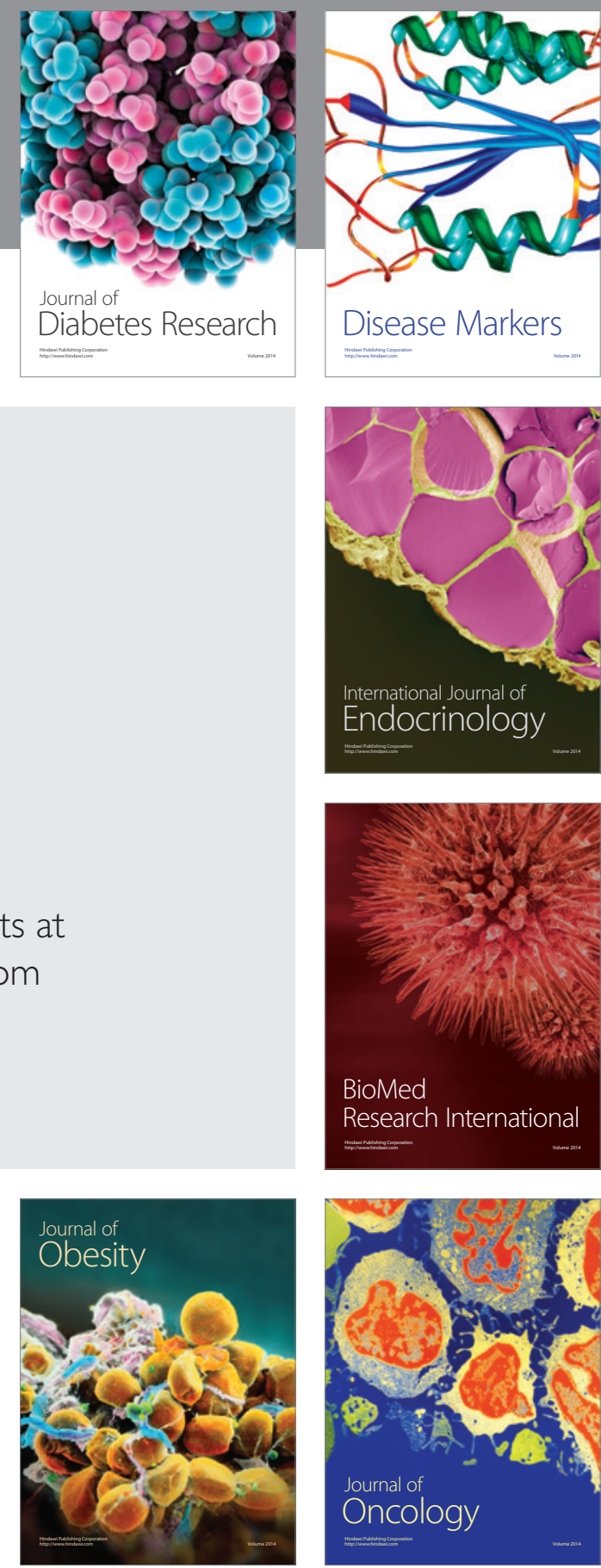

Disease Markers
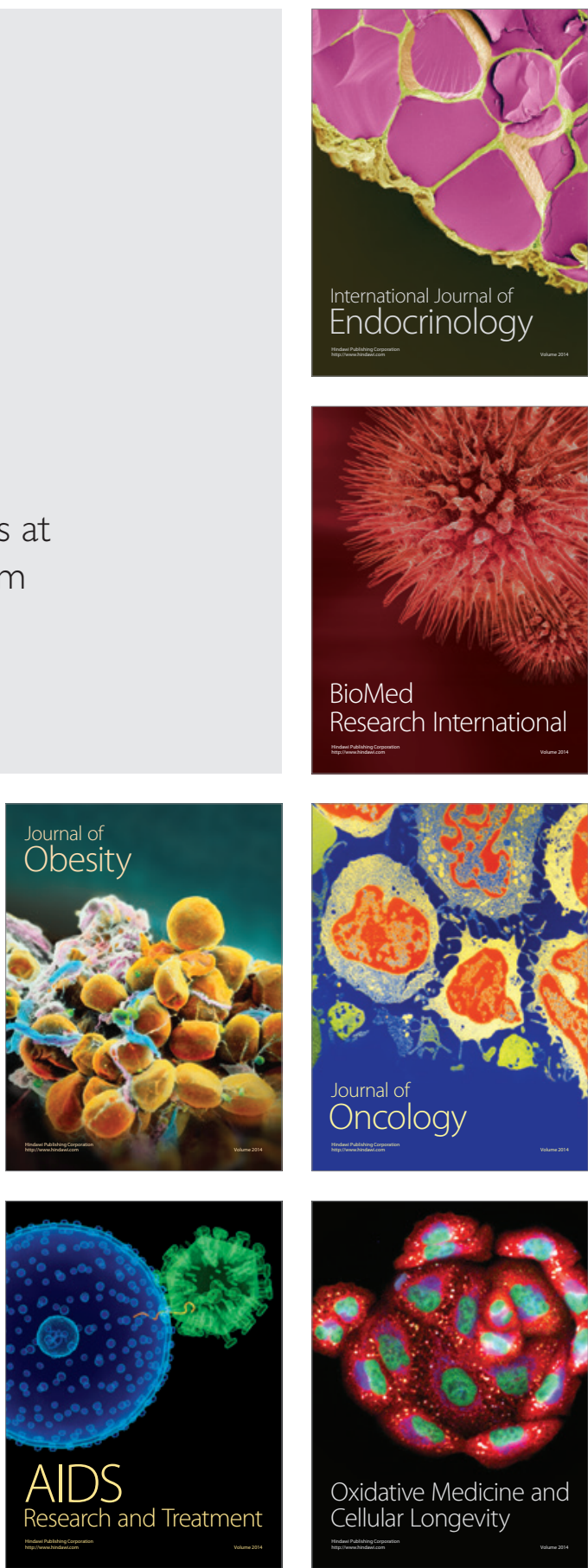